\title{
Causalidad de los incendios forestales en Pinar del Río, Cuba (1975-2018)
}

\author{
Causality of forest fires in Pinar del Río, Cuba (1975-2018)
}

\begin{abstract}
Mailyvis Ynouye-Francés ${ }^{(\mathbb{D})}$, Marcos Pedro Ramos-Rodríguez ${ }^{2 *}{ }^{(\mathbb{D})}$, Luis Wilfredo Martínez-Becerra ${ }^{3}$, Jesús María Cabrera-Reina ${ }^{\mathbb{D}}$, Raúl González-Rodríguez ${ }^{{ }^{\mathbb{D}}}{ }{ }^{\text {, Armando Duany-Dangel }}{ }^{\mathbb{1}}$

Ynouye-Francés, M., Ramos-Rodríguez, M. P., Martínez-Becerra, L. W., Cabrera-Reina, J. M., González-Rodríguez, R. y Duany-Dangel, A. (2021). Causalidad de los incendios forestales en Pinar del Río, Cuba (1975-2018). Colombia Forestal, 24(2), 24-38.
\end{abstract}

Recepción: 24 de agosto 2020

\section{Resumen}

Comprender la evolución de las causas de los incendios permite realizar un trabajo objetivo de prevención. Esta investigación analiza la causalidad de los incendios forestales en Pinar del Río, Cuba (19752018). Los análisis se realizaron considerando sub-periodos, meses del año y vegetación afectada. En el periodo ocurrieron 2896 incendios y se quemaron 51217.75 hectáreas. Los incendios originados por causas antropogénicas, principalmente las negligencias, mostraron una tendencia al aumento en el tiempo, contrario a lo ocurrido para el caso de los rayos. Esta evolución determinó que la época de incendios cambiara de marzo a junio en el sub-periodo 1975-1985, y pasara a presentarse de marzo a mayo en el sub-periodo 2008-2018. No obstante, la época donde más incendios ocurren por cada causa de forma individual no cambió durante los 44 años analizados. Estos resultados permitirán perfeccionar el trabajo de prevención de incendios forestales.

Palabras clave: época de incendios, incendios de vegetación, manejo del fuego, prevención de incendios.
Aprobación: 7 de abril 2021

\begin{abstract}
Understanding the evolution of the causes of fires allows for objective prevention work. This research aimed to analyzing the causality of forest fires in Pinar del Río, Cuba (1975-2018). The analyzes were carried out considering sub-periods, months of the year and affected vegetation. In the period, 2896 fires occurred and 51217.75 hectares were burned. The fires originated by anthropogenic causes, mainly negligence, showed an increasing trend over time, contrary to what happened in the case of lightnings. This evolution determined that the fire season changed from March to June in the 19751985 sub-period to occur from March to May in the 2008-2018 sub-period. However, the time when more fires occur for each individual cause did not change during the 44 years analyzed. These results will make it possible to improve the forest fire prevention work.
\end{abstract}

Keywords: fire season, vegetation fires, fire management, fire prevention.

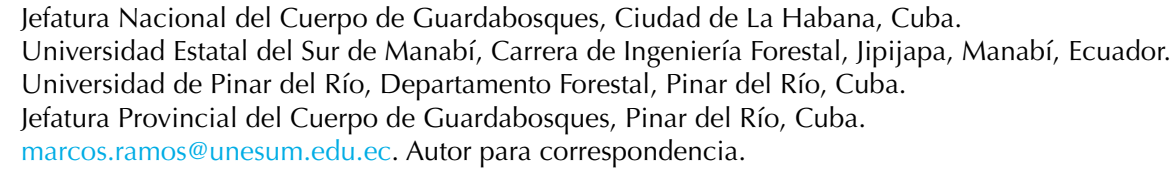




\section{INTRODUCCIÓN}

El clima y el fuego son los conductores primarios de la distribución de especies vegetales (Stroh et al., 2018). El fuego es una perturbación importante que influye en la composición de las especies, la estructura de la comunidad y la función del ecosistema en los bosques (Huang et al., 2019). No obstante, el cambio climático está aumentando la sequía y la actividad de incendios en muchas regiones propensas a estos (Sparks et al., 2018), provocando que los patrones de incendios forestales se vean alterados, probablemente (Wu et al., 2020). El fuego es un elemento intrínseco de muchos ecosistemas forestales: da forma a sus procesos ecológicos, determina la composición de especies e influye en la estructura del paisaje; sin embargo, los incendios forestales pueden tener efectos indeseables sobre la biodiversidad y la cobertura vegetal, producir emisiones de carbono a la atmósfera, liberar humo que afecte la salud humana, y provocar la pérdida de vidas y bienes. Es por ello que ha habido una creciente preocupación por los posibles impactos de la variabilidad y el cambio climático en los incendios forestales, pues este puede alterar los factores que influyen en la aparición de incendios, la disponibilidad y la inflamabilidad del combustible (Herawati et al., 2015).

Según Abatzoglou y Williams (2016) y Romps et al. (2014), en el caso de Estados Unidos estos aumentos han sido causados, en parte, por las prácticas históricas de extinción de incendios y los cambios en el uso de la tierra, aunque el cambio climático también juega un importante papel, por ejemplo, en las sequías, la presencia de temperaturas altas y el aumento de rayos. En este sentido Abatzoglou y Williams (2016) plantean que en el oeste de los Estados Unidos el cambio climático ha duplicado el área quemada por los incendios forestales durante las últimas tres décadas, en comparación con lo que se esperaría basado solo en la variabilidad natural del clima, y se han producido nueve días adicionales de alto potencial de incendios por año en los últimos 15 años. En este contexto, Flannigan et al. (2013) afirman que en Canadá las áreas forestales quemadas se duplicarán para 2100, y se prevé que cada temporada de incendios dure 20 días más en promedio. Wu et al. (2020), al desarrollar una investigación con el objetivo de explorar patrones espaciales y temporales a escala nacional de ocurrencia de incendios bajo el cambio climático actual y futuro en China, encontraron que, en general, los incendios futuros proyectados bajo escenarios de cambio climático tenían distribuciones similares (incendios ubicados principalmente en el sur y noreste de China) en comparación con la línea de base, pero con valores incrementados de probabilidad de ocurrencia de incendios.

Las causas de los incendios incluyen personas que encienden fuegos con el fin de limpiar tierras para el cultivo, aprovechar la miel de las colmenas, hacer carbón, cocinar o tratar de mantener el calor, e incendios provocados y como una forma de resolver disputas (Chinamatira et al., 2016). La temperatura, la humedad relativa, las especies arbóreas, la distancia a las vías de acceso (desde la tierra agrícola y desde el asentamiento), la velocidad del viento, la cantidad de área quemada y el mes, son los factores de riesgo que pueden afectar la ocurrencia de estos incendios (Sevinc et al., 2019). $\mathrm{Si}$ bien las causas naturales son responsables de muchos de ellos alrededor del mundo, la mayoría son causados por el hombre (Hesseln, 2018). En Estados Unidos, por ejemplo, los incendios causados por el hombre ocupan un nicho ambiental caracterizado por una menor frecuencia de rayos y una mayor humedad del combustible que los incendios causados por rayos (Balch et al., 2017).

Las causas de estos incendios varían de región a región. Para tener una estadística confiable es necesario investigar con ahínco las causas de los incendios y mantener un archivo ordenado a lo largo de los años (Soares y Batista, 2007). Es muy importante que los responsables por el combate a los incendios siempre se empeñen en descubrir y registrar la causa real o más probable del mismo (Soares et al., 2017). La identificación de patrones 
espacio-temporales de ocurrencia de incendios a escalas regionales puede brindar información valiosa para optimizar la asignación de recursos en las estrategias de extinción de incendios forestales (Carmel et al., 2009). El conocimiento de las causas de los incendios forestales y de los principales factores de ignición es un paso indispensable hacia políticas efectivas de prevención de incendios. Las causas de los incendios forestales son variadas y su distribución difiere entre países, pero también pueden diferir espacial y temporalmente dentro del mismo país (Ganteaume et al., 2013). Un buen conocimiento de los patrones espacio-temporales de las causas de la ignición de incendios forestales es crucial para una política de incendios eficaz (Curt et al., 2016). La efectividad de la prevención se basa en el conocimiento sobre la probabilidad espacio-temporal de ocurrencia de incendios y si los incendios son el resultado de procesos naturales o influencia humana. Tal conocimiento se puede usar para desarrollar herramientas y estrategias más efectivas que reduzcan tanto la probabilidad de daños como los incendios no deseados (Hesseln, 2018). En Mesoamérica las actividades humanas representan la principal causa de la ocurrencia de incendios, que se deben principalmente a situaciones de inequidad social, problemas en la tenencia de la tierra, limitados niveles culturales y de información, y políticas gubernamentales mal orientadas o desconocidas, así como propuestas que con frecuencia están fuera de un contexto real (Julio-Alvear, 2009). Según el mismo autor, en los países del Caribe es probable que la causalidad radique principalmente en quemas destinadas a limpiar terrenos para la agricultura y los asentamientos humanos, las negligencias o descuidos, la intencionalidad para la resolución de conflictos y, también, los fenómenos naturales.

El fenómeno de incendios de las coberturas vegetales es multicausal, altamente dinámico en tiempo y espacio, y generador de impactos de alcance variable sobre el medio natural y social (Jiménez et al., 2016). Teniendo en cuenta este planteamiento y los antecedentes anteriores, se desarrolló esta investigación con el fin de responder la pregunta: ¿cuál ha sido el comportamiento de la causalidad de los incendios forestales en la provincia de $\mathrm{Pi}$ nar del Río durante el periodo 1975-2018? El trabajo abordó un periodo de 44 años (1975-2018), dividido en cuatro sub-periodos de 11 años cada uno. También se consideraron causas naturales (rayos), antropogénicas (negligencia e intencionales) y desconocidas.

\section{MATERIALES Y MÉTODOS}

\section{Caracterización del área de estudio}

Cuba es la mayor isla de las Antillas, se encuentra situada en el Mar Caribe, a la entrada del Golfo de México, y constituye la porción más occidental de las Antillas Mayores. Dicha isla tiene una superficie aproximada de $110992 \mathrm{~km}^{2}$. La provincia de Pinar del Río, la más occidental de la isla de Cuba de acuerdo con la Oficina Nacional de Estadística e Información (ONEI, 2019, 2020), ocupa el cuarto lugar en extensión entre las provincias con $8884.51 \mathrm{~km}^{2}$ (8815.27 km² de tierra firme), representando el $8.09 \%$ de la superficie total del país. Geográficamente está ubicada en la región occidental, entre $21^{\circ} 19^{\prime}$ y $22^{\circ} 56^{\prime}$ de latitud norte y los $84^{\circ} 57^{\prime}$ y $83^{\circ} 05^{\prime}$ de longitud oeste. Limita al norte con el Golfo de México, al sur con el Mar Caribe, al este con la Provincia de Artemisa y al oeste con el Canal de Yucatán. La población residente es de 585555 habitantes con una densidad de 65.9 hab. $\mathrm{km}^{-2}$.

Pinar del Río tiene un clima tropical. En comparación con el invierno, los veranos tienen mucha más Iluvia. El clima se clasifica como Aw por el sistema Köppen-Geiger. La temperatura media anual es de $24.9^{\circ} \mathrm{C}$. El mes más caluroso del año es julio con un promedio de $27.5^{\circ} \mathrm{C}$, siendo febrero el más frío del año con $21.8^{\circ} \mathrm{C}$ en promedio. En un año, la precipitación promedio es de $1353 \mathrm{~mm}$. El mes más seco es diciembre, con 36 mm como promedio. En junio la precipitación alcanza su pico, 
con un promedio de $228 \mathrm{~mm}$. La diferencia en la precipitación entre el mes más seco y el mes más lluvioso es de $192 \mathrm{~mm}$. La variación en la temperatura anual está alrededor de $5.7^{\circ} \mathrm{C}$ (Climate-Data. org., 2020).

La provincia de Pinar del Río tiene un patrimonio forestal de 461197.60 ha, de las cuales están cubiertas de bosques 403741.50 ha, lo cual representa un índice de boscosidad del $45.36 \%$ si se incluye la superficie acuosa dentro del área geográfica y $47.45 \%$ excluyendo esa superficie. Los bosques naturales ocupan un área de 289437.70 ha y las plantaciones establecidas 114303.80 ha, siendo la superficie deforestada de 18212.10 ha y la inforestal de 27945.90 ha. Existen además 9226.70 ha de plantaciones jóvenes. Los bosques de Pinus sp. ocupan 129260.50 ha. Del total del patrimonio forestal el $84.42 \%$ está bajo la administración del Grupo Agroforestal, el 5.07\% en el Establecimiento Provincial para la Protección de la Flora y Fauna, el 3.60\% en las Empresas Tabacaleras, el $2.32 \%$ en el Sector Cooperativo y Campesino, y un $4.59 \%$ en otras entidades (Ministerio de la Agricultura, 2018).

\section{Frecuencia de incendios y área quemada de 1975 a 2018}

La frecuencia de incendios y área quemada se analizó durante el periodo 1975-2018 y en cuatro sub-periodos: 1975-1985, 1986-1996, 1997-2007 y 2008-2018. También se analizó desde el punto de vista espacial considerando cuatro tipos de vegetación: Pinus sp., Eucalyptus sp., Casuarina sp. y otras latifoliadas.

\section{Comportamiento periódico, estacional y espacial de las causas}

La evaluación del comportamiento periódico, estacional y espacial de las causas de los incendios forestales se desarrolló en un periodo de 44 años (1975-2018), dividido en cuatro sub-periodos de 11 años cada uno, considerando las recomendaciones de Ramos (2010), en las que se realizan evaluaciones del comportamiento histórico de los incendios forestales en lapsos de 10 años. Esto, debido a que periodos muy grandes pueden cambiar la forma de recoger las estadísticas y los métodos para la identificación de las causas. También se consideraron cuatro tipos de causas: rayos, negligencias, intencionales y desconocidas, con base en la clasificación de Ramos et al. (2009). En todos los casos se tuvieron en cuenta los elementos metodológicos desarrollados por Ramos (1999), según los cuales debe encontrarse el porqué de la ocurrencia de los incendios en un contexto espacio-temporal. En este caso los análisis se hicieron valorando los cuatro sub-periodos, los meses del año y los diferentes tipos de vegetación.

\section{Índice de causalidad y media del área quemada por incendio}

La determinación del índice de causalidad se realizó con la ecuación 1 (Vélez, 2009).

$$
I c=\frac{1}{a} \sum_{1}^{a} \frac{\sum_{1}^{a} \text { c.nic }}{n i}
$$

Donde:

Ic: índice de causalidad; c: coeficiente de peligrosidad específica de cada causa; nic: número de incendios de cada causa en cada año; ni: número de incendios en cada año; a: número de años.

La peligrosidad específica de cada causa puede medirse por la eficacia para incendiar que cada una tiene, considerando al bosque en condiciones iguales para todas ellas. Los coeficientes de cada causa fueron: 10 para intencionales, 5 para las negligencias y 1 para rayos y desconocidas, los cuales han sido fundamentados por Ramos (1999) para la provincia de Pinar del Río. La escala de peligrosidad establece que valores menores a 1 indican peligrosidad muy baja, de 1 a 2.9 baja, de 3 a 4.9 moderada, de 5 a 6.9 alta y de 7 a 10 grave. La media del área quemada por incendio es el resultado de la división del número de hectáreas 
quemadas entre el número de incendios y es un indicador que expresa la eficiencia del combate.

\section{Obtención y análisis de datos}

Los datos relacionados con los incendios forestales referentes al periodo entre enero de 1975 y diciembre del 2018 fueron facilitados por la Jefatura Provincial del Cuerpo de Guardabosques (CGB) de Pinar del Río. El procesamiento de la base de datos de incendios forestales de Pinar del Río se realizó con el Sistema Integrado para el Manejo de Bases de Datos sobre Incendios Forestales (SIMBDIF) versión 1.2 (Ramos 2002) y el análisis estadístico con el programa SPSS Statistics for Windows (version 22.0) (IBM Corp., 2013). Se trabajó con un nivel de significancia del $0.05(P=0.05)$ excepto para el análisis de regresión en el que se utilizó un nivel de significancia del $0.01(P=0.01)$. La normalidad de los datos fue verificada con la prueba estadística de Shapiro-Wilks. En todos los casos, cuando los datos se ajustaron a la distribución normal, la diferencia entre las medias se probó con un análisis de varianza, empleando para la comparación de medias la prueba de Tukey al $5 \%$. Cuando la variable dependiente no se ajustó a la distribución normal, la diferencia entre las medias fue realizada con la prueba no paramétrica de Kruskal-Wallis, comparándose los pares de medias con el empleo de la prueba post hoc de Dunn al 5\%.

\section{RESULTADOS}

\section{Frecuencia de incendios y área quemada de 1975 a 2018}

En la provincia de Pinar del Río, durante el periodo de 1975 a 2018, ocurrió un total de 2896 incendios y se quemaron 51217.75 ha. No se observan tendencias para los valores totales anuales de incendios y de áreas quemadas, en ambos casos altamente variables, destacando que solo en nueve de los 44 años del análisis el área quemada sobrepasó el promedio (1164.04 ha \pm 1992.35; Figura 1) debido a la ocurrencia durante esos años de grandes incendios que afectaron bosques de Pinus sp. y Eucalyptus sp. El promedio anual de incendios para el período de estudio fue de 65.61 \pm 26.50 ocurrencias al año. Con respecto al tipo de vegetación, el promedio anual fue de 972.50, 100.91, 14.30 y 795.74 ha quemadas para Pinus sp., Eucalyptus sp., Casuarina sp. y otras latifoliadas, respectivamente. El $94.98 \%$ de la superficie quemada en el periodo analizado corresponde a zonas de Pinus sp. y Eucalyptus sp. En cuanto a los sub-periodos 1975-1985, 1986-1996, 1997-2007 y 2008-2018 ocurrieron en ellos 509, 707, 790 y 890 incendios, respectivamente; a la vez que se quemaron 4719.23, 6099.46, 22278.22 y 18 120.84 ha, respectivamente. De un sub-periodo a otro aumentó tanto la cantidad de incendios como de hectáreas quemadas, con la excepción del último sub-periodo en el que hubo una disminución para el caso de las áreas quemadas con respecto al sub-periodo que le antecedió.

\section{Comportamiento periódico de las causas}

Al analizar la tendencia de las ocurrencias, según la causalidad durante el periodo 1975-2018 en Pinar del Río, los incendios originados por negligencias mostraron un aumento significativo en el tiempo $(r=0.69 ; P<0.01)$. Comportamiento similar mostró la intencionalidad $(r=0.34 ; \mathrm{P}<0.01)$ aunque con valores menores. Por su parte, las causas desconocidas mostraron una notable disminución $(r=-0.49 ; \mathrm{P}<0.01)$ y los rayos mostraron una leve tendencia a la disminución en el tiempo $(r=-0.05 ; P<0.01 ;$ Figura 2).

Las ocurrencias de incendios para cada una de las causas en los cuatro sub-periodos mostraron variación (Tabla 1), lo cual fue comprobado en el caso de los tres primeros sub-periodos a través de la prueba estadística no paramétrica de Kruskal-Wallis $\left(\chi^{2}=31.399 ; p=7.005 \times 10^{-7}\right.$ en el primer sub-periodo, $\chi^{2}=27.468 ; p=5.000 \times 10^{-6}$ para el segundo 


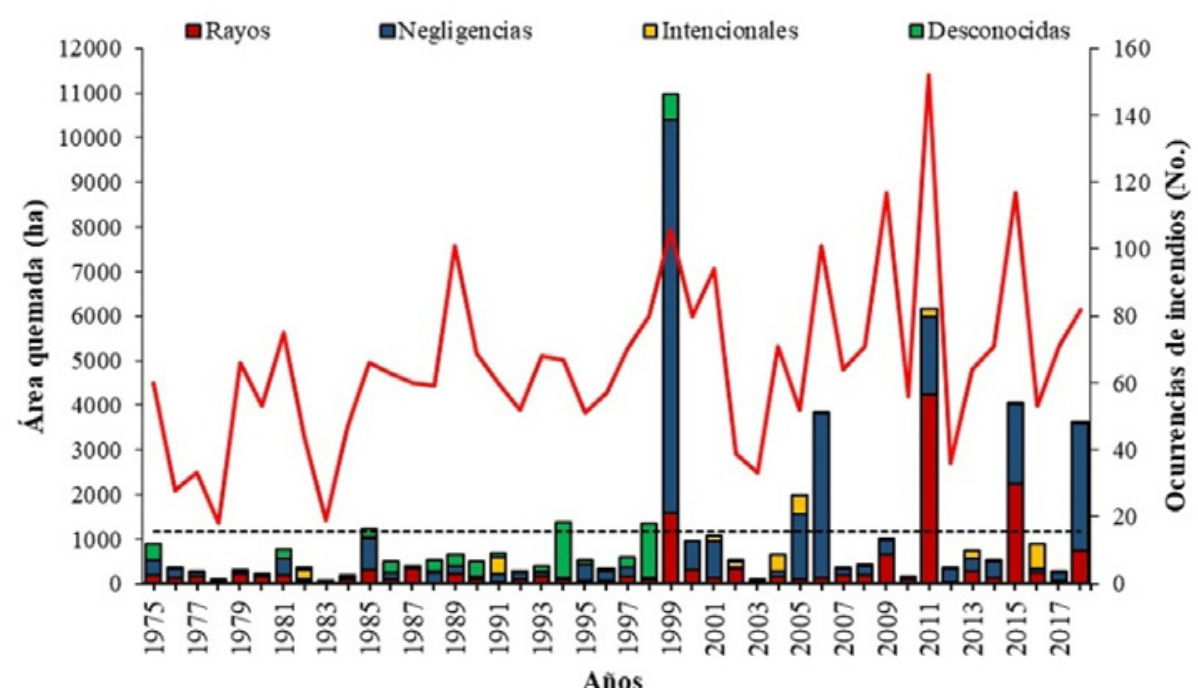

Figura 1. Número de incendios (línea continua) y área quemada (barras) en la provincia de Pinar del Río (1975-2018).

Nota: La línea segmentada indica la media anual de superficie afectada (1164.04 ha.año-1) para el período.
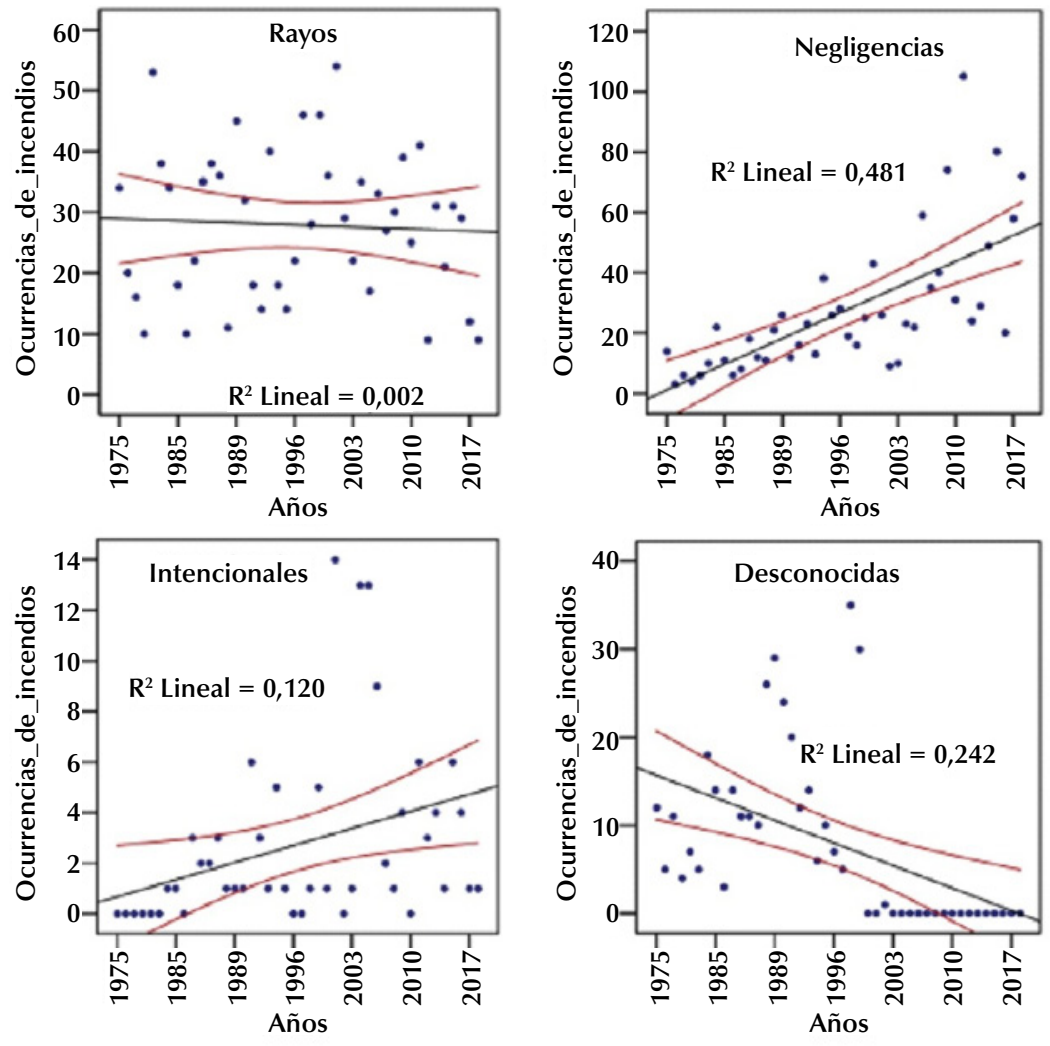

Figura 2. Tendencias principales por causas de incendios en Pinar del Río (1975-2018). 
sub-periodo y $\chi^{2}=25.243 ; p=1.400 \times 10^{-5}$ para el tercero), con un análisis de varianza en el caso del cuarto sub-periodo $\left(F=23.769 ; p=6.518 \times 10^{-7}\right)$ en el que no se consideraron las causas desconocidas pues en este no existieron. En cuanto a una misma causa, a través de los cuatro sub-periodos el análisis de varianza mostró que en el caso de los rayos no hubo diferencias entre las medias $(F=1.234$; $p$ $=0.310)$, lo cual no fue así para el caso de las negligencias $\left(F=13.803 ; p=2.521 \times 10^{-6}\right)$. Según la prueba no paramétrica de Kruskal-Wallis, también fueron diferentes las medias obtenidas para cada sub-periodo en el caso de las causas intencionales $\left(\chi^{2}=9.992 ; p=0.019\right)$ y desconocidas $\left(\chi^{2}=9.413\right.$; $\mathrm{p}=0.009)$. En este último caso no se consideró el cuarto sub-periodo.

\section{Comportamiento estacional de las causas}

Con respecto a las estaciones o épocas de incendios en Pinar del Río de 1975 a 2018 para cada una de las causas en los diferentes sub-periodos, la distribución de frecuencias a lo largo del año mostró que los incendios debidos a causas antrópicas (negligencias e intencionales) y desconocidas son significativamente más frecuentes durante los últimos meses del periodo poco lluvioso (noviembre- abril) con un máximo en abril, mientras que los incendios originados por rayos son más frecuentes durante los primeros meses del periodo lluvioso (mayo-octubre) con un valor máximo en julio, excepto en el último sub-periodo que el máximo se presentó en mayo con un pico secundario en julio.

En el caso de los rayos y las negligencias la prueba estadística no paramétrica de Kruskal-Wallis (Tabla 2) mostró diferencias entre las medias mensuales en los cuatro sub-periodos. Según los resultados de la prueba de Dunn, los meses cuyas medias de incendios originados por rayos fueron similares $y$ a la vez superiores a las de otros meses fueron mayo, junio, julio y agosto, mientras que las negligencias se concentraron en los cuatro sub-periodos durante los meses de febrero, marzo, abril y mayo, con la característica de que en los dos últimos sub-periodos los valores medios obtenidos para enero fueron similares a los determinados para alguno de los meses mencionados anteriormente. En el caso de los incendios originados de forma intencional, la prueba de Kruskall-Wallis demostró que en el primer sub-periodo no existió diferencia estadística significativa entre las medias, mientras que en los otros sub-periodos si existió diferencia estadística.

Tabla 1. Distribución de las ocurrencias de incendios según las causas y los sub-períodos objeto de estudio, sus valores medios \pm desviación estándar (ds) con la respectiva comparación de medias de acuerdo a las pruebas de Dunn (sub-periodos 1-3; intencionales y desconocidas) y Tukey (sub-periodo 4; rayos y negligencias), y los porcentajes a través de los sub-periodos en Pinar del Río (1975-2018).

\begin{tabular}{|c|c|c|c|c|c|c|c|c|c|c|c|c|}
\hline \multirow{2}{*}{ Causas } & \multicolumn{3}{|c|}{ 1975-1985 } & \multicolumn{3}{|c|}{ 1986-1996 } & \multicolumn{3}{|c|}{ 1997-2007 } & \multicolumn{3}{|c|}{ 2008-2018 } \\
\hline & (No.) & (media $\pm d s)$ & $(\%)$ & (No.) & $($ media $\pm d s)$ & $(\%)$ & (No.) & (media $\pm d s)$ & $(\%)$ & (No.) & $($ media $\pm d s)$ & $(\%)$ \\
\hline Rayos & 290 & $26.36 \pm 13.44^{a(x)}$ & 56.97 & 288 & $26.18 \pm 12.22^{a(x)}$ & 40.74 & 373 & $33.91 \pm 11.12^{a(x)}$ & 47.22 & 277 & $25.18 \pm 11.23^{a(x)}$ & 31.12 \\
\hline Negligencias & 108 & $9.82 \pm 6.01^{b(x)}$ & 21.22 & 226 & $20.55 \pm 8.60^{a(x)}$ & 31.97 & 287 & $26.09 \pm 14.76^{\mathrm{a}(x)}$ & 36.33 & 582 & $52.91 \pm 27.26^{b(y)}$ & 65.39 \\
\hline Intencionales & 7 & $0.64 \pm 1.02^{c(x)}$ & 1.38 & 24 & $2.18 \pm 1.88^{b(y)}$ & 3.39 & 59 & $5.36 \pm 5.74 \mathrm{~b}(y)$ & 7.47 & 31 & $2.82 \pm 2.13 c(y)$ & 3.48 \\
\hline Desconocidas & 104 & $9.45 \pm 4.92^{b(x)}$ & 20.43 & 169 & $15.36 \pm 8.01^{\mathrm{a}(\mathrm{x})}$ & 23.90 & 71 & $6.45 \pm 13.01^{b(y)}$ & 8.99 & 0 & $0.00 \pm 0.00$ & 0.00 \\
\hline Totales & 509 & & 100.00 & 707 & & 100.00 & 790 & & 100.00 & 890 & & 100.00 \\
\hline
\end{tabular}

Nota: ${ }^{\mathrm{a}-\mathrm{c}}$ medias en las columnas con la misma letra son estadísticamente iguales $(\mathrm{P}<0.05){ }^{(x-y)}$ medias en las filas con la misma letra entre paréntesis son estadísticamente iguales $(\mathrm{P}<0.05)$. 
Aunque estas causas son más difíciles de enmarcar en unos pocos meses, la mayoría ocurre en el primer semestre del año. Los valores medios mensuales de las causas desconocidas fueron estadísticamente diferentes para los dos primeros sub-periodos e iguales para el tercero (Tabla 2). El cuarto sub-periodo no se analizó pues en el mismo no se reportaron incendios por causas desconocidas, que tienen un comportamiento similar al mostrado por negligencias e intencionales. Esto ocurre porque todas ellas son, o bien negligencias, o bien intencionales, asociadas a las actividades humanas. Los rayos dejan una evidencia física fácil de identificar y ocurren en otra época. Como se ha visto, los meses en que habitualmente se presentan los incendios originados por cada una de las causas no han cambiado en el tiempo, sin embargo, sí lo hicieron los meses de mayor cantidad de incendios: si en el primer sub-periodo (1975-1985) la estación de incendios ocurría durante los meses de marzo a junio, en el último sub-periodo (2008-2018) esto se presentó de marzo a mayo.

\section{Comportamiento espacial de las causas}

En el caso de las ocurrencias de incendios originados por las diferentes causas, según el tipo de vegetación en cada uno de los sub-periodos objeto de estudio, la prueba estadística no paramétrica de Kruskal-Wallis (Tabla 3) mostró que para los rayos y las negligencias existen diferencias estadísticas entre las medias de incendios de cada tipo de vegetación en los cuatro sub-periodos. La misma prueba demostró que las medias de los incendios originados en cada tipo de vegetación por causas intencionales en el sub-periodo1975-1985 fueron estadísticamente similares, contrario al resto de sub-periodos que fueron estadísticamente diferentes. En los dos primeros sub-periodos las causas desconocidas mostraron medias diferentes para cada tipo de vegetación, de acuerdo a la prueba no paramétrica de Kruskal-Wallis, mientras que en el tercer sub-periodo (1997-2007) no hubo diferencias estadísticas entre las medias. Siguiendo la prueba de Dunn, la mayor cantidad de incendios producidos por rayos correspondió al Pinus sp. en todos los sub-periodos, mientras que, con respecto a las negligencias durante los dos primeros sub-periodos, los valores más altos de incendios se registraron en los bosques de Pinus sp., y en el último sub-periodo la mayor cantidad se registró en áreas con otras latifolias. En todos los sub-periodos la mayor cantidad de incendios originados tanto de forma intencional como por causas desconocidas fue reportada en las áreas de Pinus sp.

De acuerdo con los resultados anteriores, los incendios originados por rayos y negligencias ocurrieron en cantidades diferentes en los distintos tipos de vegetación durante los cuatro sub-periodos.

Tabla 2. Resultados de la prueba no paramétrica de Kruskal-Wallis para la diferencia de las medias mensuales en las ocurrencias de incendios originados por las diferentes causas, durante cada uno de los sub-periodos analizados en Pinar del Río (1975-2018).

\begin{tabular}{|c|c|c|c|c|c|c|c|c|}
\hline \multirow{2}{*}{ Causas } & \multicolumn{2}{|c|}{ 1975-1985 } & \multicolumn{2}{|c|}{ 1986-1996 } & \multicolumn{2}{|c|}{ 1997-2007 } & \multicolumn{2}{|c|}{ 2008-2018 } \\
\hline & $\left(\chi^{2}\right)$ & (p valor) & $\left(\chi^{2}\right)$ & (p valor) & $\left(\chi^{2}\right)$ & (p valor) & $\left(\chi^{2}\right)$ & (p valor) \\
\hline Rayos & 87.426 & $5.315 \times 10^{-14}$ & 82.937 & $3.980 \times 10^{-13}$ & 89.009 & $2.606 \times 10^{-14}$ & 72.404 & $4.255 \times 10^{-11}$ \\
\hline Negligencias & 55.676 & $5.826 \times 10^{-8}$ & 79.226 & $2.082 \times 10^{-12}$ & 79.762 & $1.640 \times 10^{-12}$ & 93.605 & $3.266 \times 10^{-15}$ \\
\hline Intencionales & 10.522 & 0.484 & 21.079 & 0.033 & 20.271 & 0.042 & 39.542 & $4.300 \times 10^{-5}$ \\
\hline Desconocidas & 56.885 & $3.493 \times 10^{-8}$ & 61.271 & $5.379 \times 10^{-9}$ & 7.662 & 0.743 & - & - \\
\hline
\end{tabular}


En el caso de las causas intencionales los valores fueron estadísticamente iguales en el primer sub-periodo, pero diferentes en los otros tres. Lo mismo ocurrió para las causas desconocidas durante los dos primeros sub-periodos. En el caso del tercer sub-periodo no hubo diferencia estadística entre las medias. Independiente de lo anterior, todas las causas se presentan en mayor cantidad en bosques de Pinus sp.

\section{Índice de causalidad y eficiencia del combate según las causas de incendios}

El índice de causalidad obtenido fue de 2.79, 3.58, 3.39 y 3.88 para los sub-periodos 1975-1985, 1986-1996, 1997-2007 y 2008-2018, respectivamente; valores que indican una peligrosidad baja para el primer sub-periodo y moderada para el resto. La media del índice de 1975 a 2018 fue de 3.41 por lo que los dos últimos sub-periodos se encuentran por encima de la media. El indicador muestra una tendencia lineal al aumento, explicada en un $74 \%$ de la varianza de los datos.

Los valores de área quemada por incendio en cada sub-periodo son muy variables. No obstante, su tendencia exponencial, aunque explicada solo en el $58 \%$ de la varianza de los datos, fue al aumento, con valores de 9.31, 7.63, 26.97 y 20.36 para los sub-periodos 1975-1985, 1986-1996, 1997-2007 y 2008-2018, respectivamente.

\section{DISCUSIÓN}

El aumento de la actividad de los incendios forestales obtenido en la provincia de Pinar del Río de 1975 a 2018 coincide con lo reportado por otros autores. Según Álvarez (2001), de 1961 a 1998 la evolución temporal en España presenta una tendencia creciente, tanto en el número de sucesos como en la superficie quemada. En México, de acuerdo con Cisneros-González et al. (2018), fuentes oficiales indican un incremento en los últimos años en la ocurrencia de incendios y en la superficie afectada. También en los bosques del noroeste del Pacífico, Estados Unidos (Westerling, 2016), han aumentado más rápidamente la frecuencia de incendios forestales y el área quemada en las dos últimas décadas. Autores como Bowman et al. (2017) y North et al. (2015) relacionan el aumento en tamaño, gravedad y frecuencia de los incendios forestales al cambio climático y de uso de la tierra, tendencias que hoy continúan.

La investigación realizada permitió evidenciar la evolución de las causas de los incendios a través del tiempo en la provincia de Pinar del Río, donde el porcentaje de incendios originados por rayos cambió de 56.97\% en el sub-periodo 1975-1985 a $31.12 \%$ en el sub-periodo 2008-2018. Por su parte, las ocurrencias originadas por negligencias cambiaron en los mismos sub-periodos de $21.22 \%$ a $65.39 \%$ y la intencionalidad de $1.38 \%$ a $3.48 \%$.

Tabla 3. Resultados de la prueba no paramétrica de Kruskal-Wallis para la diferencia de las medias de cada tipo de vegetación en las ocurrencias de incendios originados por las diferentes causas, durante cada uno de los subperiodos analizados en Pinar del Río (1975-2018).

\begin{tabular}{ccccccccc}
\hline \multirow{2}{*}{ Causas } & \multicolumn{2}{c}{$\mathbf{1 9 7 5 - 1 9 8 5}$} & \multicolumn{2}{c}{$\mathbf{1 9 8 6 - 1 9 9 6}$} & \multicolumn{2}{c}{$\mathbf{1 9 9 7 - 2 0 0 7}$} & \multicolumn{2}{c}{$\mathbf{2 0 0 8 - 2 0 1 8}$} \\
\cline { 2 - 8 } & $\left(\boldsymbol{\chi}^{\mathbf{2}}\right)$ & $\mathbf{( p ~ v a l o r})$ & $\left(\boldsymbol{\chi}^{\mathbf{2}}\right)$ & $\mathbf{( p \text { valor } )}$ & $\left(\boldsymbol{\chi}^{\mathbf{2}}\right)$ & $\mathbf{( p \text { valor} )}$ & $\left(\boldsymbol{\chi}^{\mathbf{2}}\right)$ & $(\mathbf{p}$ valor) \\
\hline Rayos & 34.575 & $1.498 \times 10^{-7}$ & 34.861 & $1.303 \times 10^{-7}$ & 30.230 & $1.000 \times 10^{-6}$ & 32.965 & $3.276 \times 10^{-7}$ \\
Negligencias & 24.018 & $2.500 \times 10^{-5}$ & 22.783 & $4.500 \times 10^{-5}$ & 25.780 & $1.100 \times 10^{-5}$ & 22.967 & $4.100 \times 10^{-5}$ \\
Intencionales & 4.102 & 0.251 & 10.112 & 0.018 & 16.467 & 0.001 & 17.937 & $4.530 \times 10^{-4}$ \\
Desconocidas & 24.486 & $2.000 \times 10^{-5}$ & 15.262 & 0.002 & 4.855 & 0.183 & - & - \\
\hline
\end{tabular}


La evolución descrita coincide con lo obtenido por Ramos et al. (2013) en la misma provincia durante el periodo 2002-2011, en el que los rayos provocaron el $39.26 \%$ de las ocurrencias de incendios, correspondiendo el resto a causas de origen humano tales como fumadores y limpieza de terrenos, entre otras. Contrario a lo obtenido en Pinar del Río para el periodo objeto de análisis, en un estudio realizado en Canadá, pese a existir diferencias entre las ecozonas respecto a las tendencias en el número anual de incendios mayores a 2 ha, quizás los patrones más destacados incluyeron la tendencia creciente en la ignición de rayos en la mayoría de las ecozonas de 1959 a 2018 y la tendencia decreciente en la ignición humana en la mayoría de las ecozonas de 1981 a 2018 (Coogan et al., 2020). En Europa cambió la causalidad de los incendios en el periodo de 1995 a 2010, disminuyendo los originados de forma intencional del $71 \%$ en el período 1995-1999 al 56\% en el período 2006-2010; los incendios por negligencia aumentaron del $22 \%$ al $33 \%$, mientras que los incendios accidentales aumentaron del $3 \%$ al $6 \%$ de los eventos con causas conocidas en los períodos mencionados (Ganteaume et al., 2013).

El hecho de que a partir del año 2002 disminuyeran los reportes de incendios originados por causas desconocidas, haciéndose cero en el sub-periodo 2008-2018, se explica por el trabajo que realizaron las brigadas de investigación de causas de incendios forestales, creadas en el año 2006, según Ramos et al. (2009). De acuerdo con el Cuerpo de Guardabosques (CGB, 2012), antes del año 2002 la determinación de las causas de los incendios forestales en Cuba se realizaba de manera intuitiva, a partir de la observación, la experiencia y los conocimientos que sobre el área quemada tuviera el investigador. Es desde ese año que, como parte del perfeccionamiento de esta actividad, se incorpora a este proceso investigativo el Método de Evidencias Físicas, experiencia que llega a Cuba a través de cursos dictados por especialistas de España y Chile. Posteriormente, se hicieron adecuaciones y se empezó a generalizar este conocimiento en todo el territorio nacional.

En Pinar del Río la evolución de las causas trajo como consecuencia un cambio en la época de incendios debido a que los rayos ocurren con mayor frecuencia durante la época lluviosa (mayo-octubre), lo que hace a esta causa más eficiente para originar incendios durante mayo y junio, meses en los cuales hay gran cantidad de combustibles muertos con bajos porcentajes de humedad. Las negligencias, por su parte, son más frecuentes durante el periodo poco lluvioso (noviembre-abril), alcanzando su mayor eficiencia para iniciar incendios durante marzo y abril, meses que están al final de la época poco Iluviosa. Este resultado se corresponde con un estudio realizado en el Distrito de Lichinga, norte de Mozambique, que muestra cómo los meses de septiembre y octubre, los últimos de la estación seca, fueron los que registraron el mayor número de incendios originados por causas antrópicas en todos los años estudiados (Mbanze et al., 2015). También en Colombia, durante el transcurso del año, los períodos en los que más ocurren incendios de la cobertura vegetal son enero-febrero-marzo y julio-agosto-septiembre, que coinciden con las fases mínimas del ciclo de lluvias de diferentes regiones del país (Pabón-Caicedo, 2011). Este comportamiento está relacionado con el bajo contenido de humedad del combustible durante la sequía, particularmente en combustibles vivos (follaje y ramas), que desencadena la senescencia de las hojas y su desprendimiento en los bosques de eucaliptos, y da como resultado un mayor número de cargas finas de combustible en la superficie, lo que puede aumentar la tasa de propagación del fuego (Ruthrof et al., 2016).

Coincidiendo con los resultados obtenidos en este trabajo y por los autores citados anteriormente con respecto a la relación de la época de incendios con la época de sequía, también en el Distrito de Lichinga, norte de Mozambique, la superposición de las ocurrencias de incendios con las variables 
meteorológicas mostró una gran influencia de estas variables en la ocurrencia de incendios, debido, principalmente, a los períodos secos prolongados, reportándose en el 2010 un retraso en la ocurrencia de incendios dada la temporada de lluvias un poco más larga (Mbanze et al., 2015). Al evaluar las ocurrencias de incendios forestales en el Estado de Paraná, Brasil, en el periodo 2005-2010, se obtuvo una fuerte correlación entre el número de ocurrencias de incendios y la precipitación (Tetto et al., 2012). Así mismo, al analizar la relación entre las precipitaciones y las áreas quemadas en Portugal durante el período 1975-1992, se encontró que las precipitaciones durante el verano producen un decrecimiento de las áreas quemadas, especialmente si estas ocurren en el comienzo de la época de incendios (Viegas y Viegas, 1994). Estudiando la relación entre variables meteorológicas e incendios forestales en Pinar del Río, Cuba, en los meses de mayor cantidad de ocurrencias de incendios y áreas quemadas se registraron los valores medio más altos de la velocidad del viento y los más bajos de la humedad relativa y de la precipitación (Ramos et al., 2017). En Monte Alegre, Brasil, y en Pinar del Río, Cuba, la época de incendios está fuertemente relacionada con la distribución de la precipitación a lo largo del año. En el caso de Monte Alegre el $45.42 \%$ de los incendios ocurrieron durante el periodo de agosto a octubre, mientras que en Pinar del Río el 56.54\% de los incendios ocurrieron en el periodo de marzo a mayo (Ramos et al., 2013). También en la región del Maule, Chile, de 1986 a 2012 la temporada de incendios inició a fines de invierno (agosto) culminando en otoño (mayo). La mayor parte de la ocurrencia de incendios $(84 \%)$ y superficie quemada (87\%) se presenta en los meses estivales de diciembre a marzo. El clima en la región está caracterizado por un período Iluvioso invernal y una estación seca de cuatro a seis meses (entre octubre y marzo) (Díaz-Hormazábal y González, 2016). De forma similar, en Londrina, Brasil, y en Pisa, Italia, de 2005 a 2014 la época de incendios se ubicó de julio a septiembre. En ambas regiones, julio y agosto se encuentran al final de la época de menores precipitaciones, las cuales comienzan en septiembre (Santos et al., 2019). En el Estado de Paraná, Brasil, de 2005 a 2010 la mayor cantidad de incendios ocurrió de junio a septiembre, asociado esto a que los menores valores medios de precipitación pluviométrica se presentaron durante los meses de mayo, junio y agosto (Tetto et al., 2012).

Con respecto al espacio, tipificado en esta investigación a través de cuatro tipos de vegetación, unas causas de incendios no evolucionaron de 1975 a 2018, mientras que otras sí. En este contexto, la mayor cantidad de incendios originados por rayos, de forma intencional y por causas desconocidas se registró en los bosques de Pinus sp., mientras que los incendios originados por negligencias pasaron de un mayor número en este tipo de vegetación durante los dos primeros sub-periodos, a otras latifolias en el último sub-periodo. No obstante, siempre el mayor porcentaje de ocurrencias se reportó en $\mathrm{Pi}$ nus sp. Coincidiendo con este resultado en Pinar del Río, Cuba, y en Monte Alegre, Brasil, durante el periodo 1998-2001 se reportaron en Pinus sp. porcentajes de $67.03 \%$ y $31.62 \%$, respectivamente, siendo la segunda especie más afectada en ambos lugares el Eucaliptus sp. (Ramos y Soares, 2004). Resultado diferente fue obtenido en la región del Maule, Chile, de 1986 al 2012, donde los incendios se originaron principalmente en zonas de pastizal, seguidas por matorrales y plantaciones de Pinus radiata D. Don. (Díaz-Hormazábal y González, 2016). También en Portugal y en España correspondió a P. pinaster (5.9\%) y Eucalyptus spp. (6.0\%) el mayor porcentaje de parcelas del Inventario Forestal Nacional quemadas para la Península Ibérica. En Portugal, el $12.0 \%$ y el $10.0 \%$ de las parcelas quemadas correspondieron a parcelas de $P$. pinaster $y$ de Eucalyptus spp., respectivamente, mientras que en España el porcentaje de parcelas quemadas para las mismas especies fue del $34 \%$ y del $11 \%$, respectivamente (Nunes et al., 2019).

El aumento del área quemada por incendio de 9.31 en el sub-periodo 1975-1985 a 20.36 ha por incendio en el sub-periodo 2008-2018, está relacionado con la ocurrencia en los últimos 22 años 
del periodo objeto de estudio de incendios que quemaron gran superficie de bosques, como los dos que en 1999 quemaron más de 11000 ha en total. En el período 1989-1996 fue reportado para Pinar del Río un valor medio de 7.79 ha quemadas por incendio (Ramos y Soares, 2004), similar al obtenido en esta investigación para el sub-periodo 19861996. Durante el período 2002-2011 la media del área quemada por incendio en Pinar del Río, Cuba, fue de 18.90, valor similar al obtenido en esta investigación para los dos últimos sub-periodos y muy superior al obtenido para Monte Alegre, Brasil, que fue de 0.46 ha por incendio (Ramos et al., 2013). En Londrina, Brasil, y Pisa, Italia, durante el periodo 2005-2014 se reportaron valores medios de 1.67 y 2.05 ha quemadas por incendio, respectivamente, (Santos et al., 2019), los cuales son inferiores a los obtenidos en esta investigación.

\section{CONCLUSIONES}

En la provincia de Pinar del Río los incendios originados por causas antropogénicas, principalmente las negligencias, mostraron una tendencia al aumento en el tiempo, contrario a lo ocurrido para el caso de los rayos. Esta evolución determinó que la época de incendios pasara de presentarse entre marzo y junio en el sub-periodo 1975-1985, a presentarse entre marzo y mayo en el sub-periodo 2008-2018. No obstante, la época y el tipo de vegetación donde más incendios ocurren por cada causa de forma individual no cambió durante los 44 años analizados. Estos resultados permitirán perfeccionar el trabajo de prevención de incendios forestales.

\section{AGRADECIMIENTOS}

Los autores agradecen a la Universidad de Pinar del Río, Cuba, al Cuerpo de Guardabosques de Cuba y a la Universidad Estatal del Sur de Manabí, Ecuador. También al editor de Colombia Forestal y a los dos evaluadores anónimos, quienes enriquecieron este trabajo con sus sabias recomendaciones, preguntas y observaciones.

\section{CONFLICTOS DE INTERÉS}

Los autores declaran no tener conflicto de intereses.

\section{CONTRIBUCIÓN POR AUTOR}

M.Y.F y M.P.R.R. idearon la investigación, realizaron el procesamiento estadístico de los datos y redactaron el documento. Todos los autores contribuyeron a la recolección de los datos y la discusión, y comentaron los borradores.

\section{REFERENCIAS}

Abatzoglou, J. T. y Williams, A. P. (2016). Impact of anthropogenic climate change on wildfire across western US forests. Proceedings of the National Academy of Sciences of the United States of America, 113(42), 11770-11775.

https://doi.org/10.1073/pnas.1607171113

Álvarez, R. Y. (2001). Evolución histórica de los incendios forestales en España. Nimbus: Revista de Climatología, Meteorología y Paisaje, (7-8), 39-49.

http://repositorio.ual.es:8080/bitstream/handle/10835/1394/251280.pdf? sequence=1 \&isAllo wed $=\gamma$

Balch, J. K., Bradley, B. A., Abatzoglou, J. T., Nagy, R. C. y Fusco, E. J. (2017). Human-started wildfires expand the fire niche across the United States. Proceedings of the National Academy of Sciences, 114(11), 2946-2951.

https://doi.org/10.1073/pnas.1617394114

Bowman, D. M. J. S., Williamson, G. J., Abatzoglou, J. T., Kolden, C. A., Cochrane, M. A. y Smith, A. M. S. (2017). Human exposure and sensitivity to globally extreme wildfire events. Nature Ecology \& Evolution, 1(February), 1-6. 
https://doi.org/10.1038/s41559-016-0058

Carmel, Y., Paz, S., Jahashan, F. y Shoshany, M. (2009). Assessing fire risk using Monte Carlo simulations of fire spread. Forest Ecology and Management, 257(1), 370-377.

https://doi.org/10.1016/j.foreco.2008.09.039

CGB. Cuerpo de Guardabosques. (2012). Guía rápida para la investigación de causas de incendios forestales [en Cuba].

Chinamatira, L., Mtetwa, S. y Nyamadzawo, G. (2016). Causes of wildland fires, associated socio-economic impacts and challenges with policing, in Chakari resettlement area, Kadoma, Zimbabwe. Fire Science Reviews, 5(1), 1-11. https://doi.org/10.1186/s40038-016-0010-5

Cisneros-González, D., Zúñiga-Vásquez, J. M. y Pompa-García, M. (2018). Actividad del fuego en áreas forestales de México a partir de sensores remotos y su sensibilidad a la sequía. Madera y Bosques, 24(3), 1-11.

https://doi.org/10.21829/myb.2018.2431687

Climate-Data.org. (2020). Clima de Pinar del Río: climograma de Pinar del Río. https://es.climate-data.org/america-del-norte/cuba/pinar-del-rio/pinar -del-rio-294/

Coogan, S. C. P., Cai, X., Jain, P. y Flannigan, M. D. (2020). Seasonality and trends in human- and lightning-caused wildfires $\geq 2$ ha in Canada, 19592018. International Journal of Wildland Fire, 29(6), 473-485.

https://doi.org/10.1071/WF19129

Curt, T., Fréjaville, T. y Lahaye, S. (2016). Modelling the spatial patterns of ignition causes and fire regime features in southern France: Implications for fire prevention policy. International Journal of Wildland Fire, 25(7), 785-796. https://doi.org/10.1071/WF15205

Díaz-Hormazábal, I. y González, M. E. (2016). Análisis espacio-temporal de incendios forestales en la región del Maule, Chile. Bosque (Valdivia), 37(1), 147-158.

https://doi.org/10.4067/S0717-92002016000100014

Flannigan, M., Cantin, A. S., De Groot, W. J., Wotton, M., Newbery, A. y Gowman, L. M. (2013). Global wildland fire season severity in the 21 st century. Forest Ecology and Management, 294, 54-61. https://doi.org/10.1016/j.foreco.2012.10.022

Ganteaume, A., Camia, A., Jappiot, M., San-MiguelAyanz, J., Long-Fournel, M. y Lampin, C. (2013). A review of the main driving factors of forest fire ignition over Europe. Environmental Management, 51(3), 651-662.

https://doi.org/10.1007/s00267-012-9961-z

Herawati, H., González-Olabarria, J. R., Wijaya, A., Martius, C., Purnomo, H. y Andriani, R. (2015). Tools for Assessing the Impacts of Climate Variability and Change on Wildfire Regimes in Forests. Forests, 6(5), 1476-1499.

https://doi.org/10.3390/f6051476

Hesseln, H. (2018). Wildland Fire Prevention: a Review. Current Forestry Reports, 4, 178-190.

https://doi.org/10.1007/s40725-018-0083-6

Huang, W., Liu, X., González, G. y Zou, X. (2019). Late Holocene fire history and charcoal decay in subtropical dry forests of Puerto Rico. Fire Ecology, 15(1). https://doi.org/10.1186/s42408-019-0033-0

IBM Corp. (2013). IBM SPSS Statistics for Windows, Version 22.0. Armonk, NY: IBM Corp.

Jiménez, A. M., Urrego, L. E. y Toro, L. J. (2016). Evaluación del comportamiento de incendios de la vegetación en el norte de Antioquia (Colombia): análisis del paisaje. Colombia Forestal, 19(2), 161-180.

https://doi.org/10.14483/udistrital.jour.colomb. for.2016.2.a03

Julio-Alvear, G. (2009). Panorámica general de los incendios forestales en Iberoamérica. En R. Vélez (Ed.), La defensa contra incendios forestales. Fundamentos y experiencias ( $2^{a}$ ed.) (pp. 803-810). McGraw-Hill/Interamericana de España, S. L.

Mbanze, A. A., Batista, A. C., Tetto, A. F., Koehler, H. S. y Manteiga, J. B. (2015). Influence of the meteorological conditions on forest fires occurrences in Lichinga District, Northern Mozambique. Floresta, 45(3), 577-586.

https://doi.org/10.5380/rf.v45i3.33742

Ministerio de la Agricultura. (2018). Dinámica forestal. Pinar del Río, Cuba. 
North, B. M. P., Stephens, S. L., Collins, B. M., Agee, J. K., Aplet, G., Franklin, J. F. y Fulé, P. Z. (2015). Reform forest fire management. Science, 349(6254), 1280-1281.

https://doi.org/10.1126/science.aab2356

Nunes, L., Álvarez-González, J., Alberdi, I., Silva, V., Rocha, M. y Rego, F. C. (2019). Analysis of the occurrence of wildfires in the Iberian Peninsula based on harmonised data from national forest inventories. Annals of Forest Science, 76(1).

https://doi.org/10.1007/s13595-019-0811-5

ONEI. Oficina Nacional de Estadística e Información. (2019). Anuario Estadístico de Pinar del Río 2018. http://www.onei.gob.cu/sites/default/files/anuario_ est_provincial/00_anuario_completo_2018.pdf

ONEI. Oficina Nacional de Estadística e Información. (2020). Cuba y sus territorios: Pinar del Río. http://www.onei.gob.cu/mapa/provincia/pinar-del -rio

Pabón-Caicedo, J. D. (2011). Los incendios de la cobertura vegetal en Colombia y su relación con la variabilidad climática y con el cambio climático. En Á. Campo Parra-Lara (Ed.), Incendios de la cobertura vegetal en Colombia Tomo I ( ${ }^{\text {a }}$ ed.) (pp. 145-165). Universidad Autónoma de Occidente.

Ramos, M. P. (1999). Bases metodológicas para el perfeccionamiento de la prevención de los incendios forestales [Tesis Doctoral, Universidad de Pinar del Río]. Repositorio institucional de la Universidad de Pinar del Río. https://rc.upr.edu.cu/jspui/bitstream/ DICT/2189/1/Marcos\%20Pedro\%20Ramos\%20 Rodr\%C3\%ADguez.pdf

Ramos, M. P. (2002). Sistema Integrado para el Manejo de Bases de Datos sobre Incendios Forestales (SIMBDIF) Versión 1.2. [Presentación de Paper]. III Congreso Forestal Venezolano, Ciudad Bolivar, Venezuela.

Ramos, M. P. (2010). Manejo del fuego ( $1^{\mathrm{a}} \mathrm{ed}$.). Editorial Félix Varela.

Ramos, M. P. y Soares, R. V. (2004). Análisis comparativo entre los incendios forestales en Monte Alegre, Brasil y Pinar del Río, Cuba. Floresta, 34(2), 101-107. http://dx.doi.org/10.5380/rf.v34i2.2379
Ramos, M. P., González, R., Figueredo, M. C. y Martínez, L. W. (2009). La defensa contra los incendios forestales en Cuba. En R. Vélez (Ed.), La defensa contra los incendios forestales. Fundamentos y experiencias ( $2^{\mathrm{a}}$ ed.) (pp. 813-821). McGraw-Hill/Interamericana de España, S. L.

Ramos, M. P., Soares, R. V., Batista, A. C., Tetto, A. F. y Martínez, L. W. (2013). Comparação entre o perfil dos incêndios florestais de Monte Alegre, Brasil, e de Pinar del Río, Cuba. Floresta, 43(2), 231-240.

http://dx.doi.org/10.5380/rf.v43i2.27650

Ramos, M. P., Carrasco, Y., Medina, C. A., Batista, A. C. y Tetto, A. F. (2017). Relación entre variables meteorológicas e incendios forestales en la provincia Pinar del Río, Cuba. Floresta, 47(3), 343-352.

Romps, D. M., Seeley, J. T., Vollaro, D. y Molinari, J. (2014). Projected increase in lightning strikes in the United States due to global warming. Science, 346(6211), 851-854.

https://doi.org/10.1126/science.1259100

Ruthrof, K. X., Fontaine, J. B., Matusick, G., Breshears, D. D., Law, D. J., Powell, S. y Hardy, G. (2016). How drought-induced forest die-off alters microclimate and increases fuel loadings and fire potentials. International Journal of Wildland Fire, 25(8), 819-830.

https://doi.org/http://dx.doi.org/10.1071/WF15028

Santos dos, J. F. L., Tetto, A. F., Bertacchi, A., Batista, A. C. y Soares, R. V. (2019). Comparison of Forest Fire Profiles in Londrina, Brazil and Pisa, Italy. Floresta e Ambiente 26(2), 1-10. https://doi.org/10.1590/2179-8087.060717

Sevinc, V., Kucuk, O. y Goltas, M. (2019). A Bayesian network model for prediction and analysis of possible forest fire causes. Forest Ecology and Management, 457, 117723. https://doi.org/10.1016/j.foreco.2019.117723

Soares, R. V. y Batista, A. C. (2007). Incêndios florestais: controle, efeitos e uso do fogo. Universidade Federal do Paraná.

Soares, R. V., Batista, A. C. y Tetto, A. F. (2017). Incêndios florestais: controle, efeitos e uso do fogo $\left(2^{\mathrm{a}}\right.$ edición revisada). Universidade Federal do Paraná. 
Sparks, A. M., Talhelm, A. F., Feltrin, R. P., Smith, A. M. S., Johnson, D. M., Kolden, C. A. y Boschetti, L. (2018). An experimental assessment of the impact of drought and fire on western larch injury, mortality and recovery. International Journal of Wildland Fire, 27(7), 490-497. https://doi.org/10.1071/WF18044

Stroh, E. D., Struckhoff, M. A., Stambaugh, M. C. y Guyette, R. P. (2018). Fire and Climate Suitability for Woody Vegetation Communities in the South Central United States. Fire Ecology, 14(1), 106-124. https://doi.org/10.4996/fireecology.140110612

Tetto, A. F., Batista, A. C. y Soares, R. V. (2012). Ocorrência de incêndios florestais no estado do Paraná, no período de 2005 a 2010. Floresta, 42(2), 391-398.

http://dx.doi.org/10.5380/rf.v42i2.22516
Vélez, R. (2009). Instrumentos de planificación: índices de predicción del riesgo. En R. Vélez (Ed.), La defensa contra los incendios forestales. Fundamentos y experiencias ( $2^{\mathrm{a}}$ ed.) (pp. 248-254). McGraw-Hill/ Interamericana de España, S. L.

Viegas, D. X. y Viegas, M. T. (1994). A relationship between rainfall and burned area for Portugal. International Journal of Wildland Fire, 4(1), 11-16.

https://doi.org/10.1071/WF9940011

Westerling, A. L. (2016). Increasing western US forest wildfire activity: sensitivity to changes in the timing of spring. Philosophical Transactions Royal Society, 371, 1-10. http://dx.doi.org/10.1098/rstb.2015.0178

Wu, Z., He, H. S., Keane, R. E., Zhu, Z., Wang, Y. y Shan, Y. (2020). Current and future patterns of forest fire occurrence in China. International Journal of Wildland Fire, 29(2), 104-119. https://doi.org/https://doi.org/10.1071/WF19039

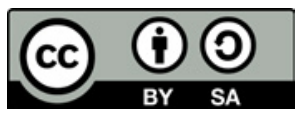

